\title{
Research of Routing Mechanism on Adaptive Wireless Sensor Networks
}

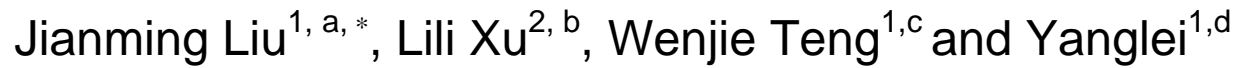 \\ ${ }^{1}$ Computer Department, Weifang Medical University, Weifang, 261053, China \\ ${ }^{2}$ Weifang Vocational College, Weifang, 261042, China

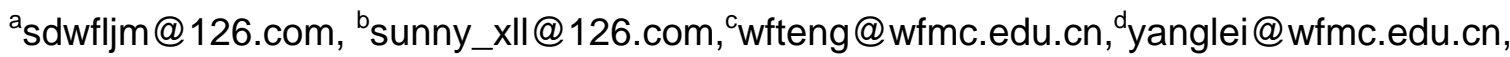 \\ ${ }^{*}$ corresponding author
}

Keywords: Wireless Sensor Networks; Routing protocol; Adaptive; Topology

\begin{abstract}
It is an important challenge to find out suitable security efficient routing mechanism for wireless sensor networks due to limitations of power. This paper has studied a mechanism based on an adaptive network node routing. The adaptive routing mechanism can make the route of nodes within the network more efficient and reasonable. At the same time, it can improve the average life of the network, and make the network data transmission more efficient and reliable.
\end{abstract}

\section{Introduction}

At present, Wireless Sensor Network (WSN) has been a hot issue and has received considerable attention for decades we all know, most sensor network is deployed in a random mode. Nodes do not have any information about neighbors and topology of the network at beginning. The computing resources of micro-sensor nodes are highly limited, which influences the design of WSN security techniques [1]. It is an important challenge to find out suitable security efficient routing mechanism for wireless sensor networks due to the limitations of power. This paper has studied a mechanism based on the adaptive network node routing. The adaptive routing mechanism can make the route of nodes within the network more efficient and reasonable.

\section{Routing Protocol for Wireless Sensor Networks}

Wireless routing network routing technology has a crucial impact on network performance. It can achieve high efficiency, low latency, and strong connectivity. In recent years, there are a lot of the research results at home and abroad. From the perspective of network topology, it is divided into two categories: plane routing protocol and clustered routing protocol [2].

Plane Routing Protocol [3]. In the network structure of the plane routing protocol, all the sensor nodes in the whole network are equal. There is no network level and level differences between the nodes. The routing information is generated by the local operation and feedback information between the nodes. The source node in the monitoring area sends the monitored sensor data to the destination node. After receiving the query command from the destination node, the source node sends the detected sensor data to the destination node.

Clustered Routing Protocols. In the network structure of the clustered routing protocol, the network is generally divided into clusters of different sizes, and the set of wireless sensor nodes is assembled according to certain rules. Each cluster consists of a cluster head node and a large number of member nodes. The low-level network cluster head node is also a member node in the high-level network cluster. The communication between the cluster head node and the external base station is completed. The specific network topology is shown in Fig.1. The clustered routing protocol algorithm divides all the nodes of the sensor nodes into several parts that are connected [4].

Under the clustering network topology mechanism, all the sensor nodes in the wireless sensor network are divided into two categories: cluster head node and member nodes. In each cluster, a node is selected as a cluster head node according to certain principles. The cluster head node manages and controls all the member nodes in the whole cluster, coordinates the work between the member nodes, collects and integrates the cluster information, and forwards inter-cluster data [5]. 


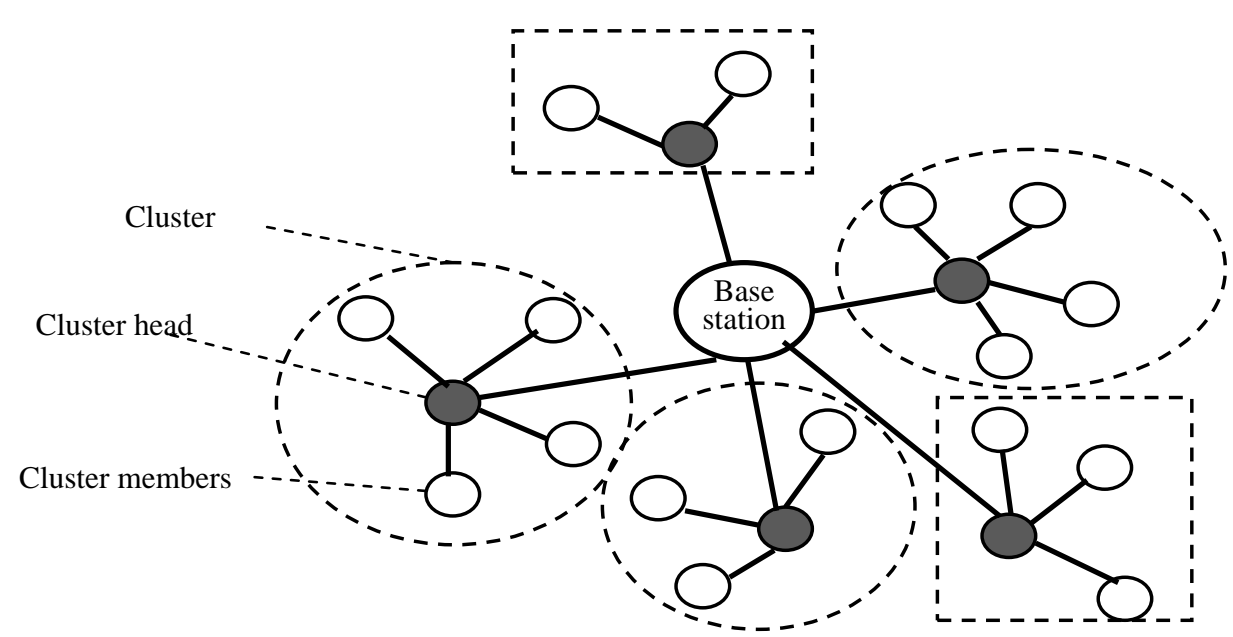

Fig1.WSNS Clustered Routing Protocol Topology

\section{Wireless Sensor Network System Model}

According to Fig. 2, the wireless sensor network model is mainly described as follows: $N$ wireless sensor nodes is randomly deployed in a two-dimensional area $F \times F$, and the external base station is deployed in the sensor area $h$ distance. Assuming that each node has only limited energy and data processing capabilities, the task of the node is to work in accordance with the instructions of the external control center, data collection, aggregation, transmission and other dynamic work. All sensor nodes and external base stations are in a stationary state [6]. The external base station uses the beacons signal to find the corresponding active node in the trigger phase of the wireless sensor network. The external base station is responsible for organizing, coordinating, managing the sensor nodes, and it can collect the monitoring data, bring together the original data and contact with the outside control center. Finally, the control center will pass the processed information to the end user.

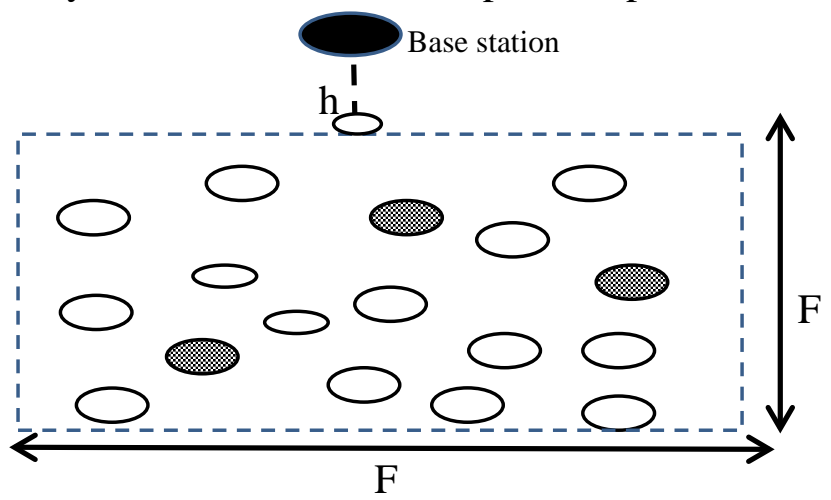

Fig 2. Wireless Sensor Network Model

In the above system model (Figure 2), it is assumed that all the sensor nodes can report the remaining energy information to the external base station. It can also switch intelligently in both the open and the sleep state. If the sensing circuit and the data processing circuit can be smart to switch, the node transmission distance can be programmed to manage. At present, Sen Tech company has developed a voice sensor module with a wireless sensor node, and the module has the above function. For any sensor node in the model, we can assume that there is a certain transmission distance between them.

According to the energy consumption model of node communication, the definition of the node to send information and receive information energy consumption formula is as follows:

Energy consumption when sending information: $E s=\left(\beta_{1}+\beta^{\prime} D_{2}\right) * M$

Energy consumption when receiving information: $E \mathrm{r}=\beta_{2} * M$

Among them: Es represents the energy consumption when the wireless sensor node sends the message; $\beta_{1}$ and $\beta_{2}$ represent the energy consumption when the wireless sensor node in the process of sending and receiving information, and the value is $50 \mathrm{~nJ} / \mathrm{bit} ; \beta^{\prime}$ represents the unit energy (bit) in 
the transmission process in the unit area $\left(\mathrm{m}^{2}\right)$ within the loss of energy, the value of take 100pJ/ $\mathrm{bit} / \mathrm{m}^{2} ; M$ represents the number of bits of information; $D$ indicates the information transmission distance.

\section{Based on the Adaptive Wireless Sensor Network Routing Mechanism}

At present, the sensor network routing structure includes hierarchical and cluster-based two categories. In order to describe the proposed routing mechanism, the following definitions are given:

Definition 1 Set the transmission radius of the common sensor node to $R$. In the actual network operation, it is possible to dynamically adjust the node transmission radius $\mathrm{R}$ according to the current residual energy of the node.

Definition 2 Set $\mathrm{N}$ sensor nodes be deployed in the monitoring area of area $S$, then the node average distribution density (Mean Node Distribution Density) is denoted as MNDD $=N / S$.

Definition 3 Set $\mathrm{s}$ be a selected sensor node in the deployment area, then the node distribution density in the circular area with $s$ as the center of the selected value $r$ is called the local node distribution density (LNDD $=n / \pi * r * r$ ) where $\mathrm{n}$ is the number of nodes in the circular area.

Definition 4 In the area of the wireless sensor network, after the node is deployed, the node is divided into two different sets according to whether the node is within the radio frequency direct transmission range of the base station. They are called the base station reachable node set and the base station unreachable node set. They are recorded as $S R$ and $S U R$.

The specific program is described as follows.

Step1. Determine the Coverage Area of the Node and the Base Station Connectivity, and Node Density Level. (1) According to whether the node is in the base station, direct transmission range within the entire node is divided into set $R_{S}$ and set $S_{U R}$; (2) Calculate the average distribution density of nodes in the deployed area MNDD; (3) The nodes are sorted according to the node energy size in the set $R_{S}$ (if the node energy is the same, it is randomly selected part of the $R_{S}$ node at the first round). The nodes with a large number of energy are used as the center and the node propagation distance as the radius. The selected nodes can cover all the nodes in the $S_{U R}$, until the last $i$ node; (4)Calculate the local node distribution density of $1 N N N, 2 L N D D, \ldots$ iLNDD without overlapping nodes in (3).

Step2. According to the Results of Step 1, the Nodes in the Coverage Area are Classified. (1) Repeat (1) to (4) of Step1 for a node set that exceeds the threshold for $\operatorname{LNDDj}(j \in[1, i])$ with a reference parameter as the threshold.

For cluster nodes, if the node energy variant $\delta\left(E_{\text {current }} \geqq \not\right.$, then go to Step 3 ; otherwise go to Step4. Where $E_{\text {current }}$ is the current residual energy of the node, $\tau$ is a predetermined threshold, and can be dynamically adjusted according to the relevant result.

Step 3. Use the Clustered Routing Protocol to Process Each Node Collection. (1) The node belonging to the $R_{S}$ in the set of nodes produces $a$ random number $r$ between 0 and 1 . If the random number $r$ is less than the defined threshold $T(n)$, the node is selected as cluster head. The formula for $T(n)$ is following:

$$
T(n)=\frac{p}{1-p[\operatorname{rmod}(1 / p)]}\left[\frac{E_{n \_ \text {current }}}{E_{n \_ \text {max }}}+\left(r_{s} \operatorname{div} \frac{1}{p}\right)\left(1-\frac{E_{n \_ \text {current }}}{E_{n \_ \text {max }}}\right)\right]
$$

(2) The cluster head node and the single node use the plane routing protocol to deal with, the high-level node through its cluster head to the lower node to transmit information, then go to Step5.

Step4. Because the Energy Difference between Nodes in the Cluster is very Small, the Routing between Nodes is done by Hop or Hop. The data transmission using a plane-based routing protocol algorithm, then go to Step5.

Step 5. Calculate the Objective Function $f(R)=\varepsilon^{*} d * N / n$. If the $B_{S}$ does not receive packets from any of the sensors within a certain time interval, adjust the node of the transmission range $R+\Delta r \rightarrow R$; otherwise the transmission range is $R+\Delta r \rightarrow R$ with the probability of $\left[1-0.5^{*}(n / N)\right]$; otherwise adjust the transmission range $R-\Delta r \rightarrow R$ with the probability of $\left[0.5^{*}(n / N)\right]$, go to Step1 at 
a certain time interval. Where $\varepsilon$ is the average energy consumption of the sensor packet, $d$ is the delay of the sensor packet, and $\Delta r$ is a predetermined value. It can be dynamically adjusted according to the correlation result.

\section{Analysis of Adaptive Route Mechanism}

In the practical application of the wireless sensor network, when the sensor node is deployed in the monitoring area, some nodes become unreachable nodes for some reasons. The adaptive routing mechanism can solve this problem by using the base station reachable node as a transmission intermediary between the unreachable node and the base station. Considering the bottleneck effect in the process of signal transmission in the region with small node density, the routing mechanism cluster processes the large density node area account of the residual energy of the nodes. The adaptability of the wireless sensor network is mainly reflected that the wireless sensor network node has a certain learning and adapt ability according to the environment and system changes and the corresponding changes in their behavior. If there is a problem with the node resource, especially the energy resource, the wireless sensor network must be able to adjust the operation strategy (such as reducing the transmission radius of the node or making the idle node in the dormant state), so as to prolong the life cycle of the network system. The adaptive mechanism of the proposed wireless sensor network based on adaptive is mainly embodied in the following four aspects:

The Adaptability of the Node Network. After the sensor network is deployed, a large number of nodes are randomly thrown into the monitoring area. Therefore, a lot of nodes cannot go through their own direct information exchange of the situation. At this time, the mechanism divides the whole node into set $R_{S}$ and set $S_{U R}$. The base station or active node can be used as an agent to relay the information of these nodes, so that these nodes can join the network as much as possible. It can expand the scale of network monitoring, improve the utilization of resources and network availability, and make the node in the process of networking have a good self-adaptability.

The Adaptability of Node Energy. In general, the energy consumption rate of nodes in the network determines the life of the network, and different methods of measuring the life of the network have different standards. At present, the universal measurement method is based on the network nodes which cannot be normal network and work when the network is declared dead. The mechanism mainly considers the following problem for the energy consumption problem of the node: For any selected node, if its energy value variance $\delta$ (E current) $\geqq$ tbetween the neighbor node and a neighbor node is within a given range. That is, $\delta$ (E current) $<\tau$, it indicates that the energy difference between the nodes is not large but balanced, so a balanced energy consumption of the routing method is used in the process of node network; When the variance of the energy value between the neighbor node and the neighbor node exceeds a given range, $\delta(E$ current $) \geqq \tau$ It indicates that the energy difference between nodes is very large, and at this time, it is suitable for the use of energy non-equilibrium consumption of the routing method. Therefore the mechanism has adaptive characteristics in terms of node energy consumption.

The Adaptability of the Node Transmission Radius. Relevant experiments show that the transmission of $1 \mathrm{~Kb}$ packets on a $100-\mathrm{m}$ communication line is almost the same as the energy consumed by the CPU to execute 3Mb. Therefore, the sensor node should focus on local data processing and reduce the long-distance data transmission. It can reduce the enormous cost of energy brought by communication. In the course of the work of the node, the average energy consumption of the sensor node and the delay of the transmission packet from the node to the base station are taken into consideration, and the objective function is: $f(R)=\varepsilon * d * N / n$. With the base station as the core, the adequacy of the radius of the transmission of the node is measured, and the transmission radius of the node can be adjusted in time if the transmission radius of the node can be adjusted: $R-\Delta r \rightarrow R$, which can reduce the unnecessary loss of energy. If the transmission radius of the node is small enough to affect the normal operation of the network, the mechanism can adjust the transmission radius of the node in time: $R+\Delta r \rightarrow R$, and it makes the network work normally. Based on this, the mechanism allows the network to operate in the most appropriate state in an adaptive state. 
Self-adapt Ability of Node Construction Routing. Considering the characteristics of the wireless sensor network, the adaptive routing mechanism takes full account of the energy consumption problem of each node in the network. The nodes in the network are at the same rate of consumption and extend the life cycle of the whole network. At the same time, the routing mechanism takes full account of the load balancing between nodes. Through the effective cooperation between nodes for data transmission and processing, it can minimize network communication overhead. Finally, the routing mechanism to fully consider the network scalability and node mobility needs. It can adapt to the dynamic changes in the network. Thus, the mechanism is adaptive in terms of node construction routing.

\section{Conclusion}

The paper has proposed an adaptive wireless sensor network routing mechanism. The mechanism can determine the routing mode of the network by the residual energy of the node itself and the clustering density of the neighboring nodes. It considers the node transmission radius adjustment and other issues. Theoretical and application analysis shows that the adaptive routing mechanism can make the route of nodes within the network more efficient and reasonable. At the same time, it can improve the average life of the network, and make the network data transmission more efficient and reliable. The further study is to work in the practical application of the full consideration of the proposed routing mechanism of the characteristics and method, to test and optimize it from the perspective of practice.

\section{Acknowledgements}

The research work was supported by the Shandong Province Medical Science and Technology Program under grant NO.2014WS0460.and supported by the Shandong Province Graduate Education Innovation Fund under grant NO. SDYY16058.

\section{References}

[1] M. A. Rahman, S. Hussain, Energy Efficient Data Dissemination for Uniform Coverage in Wireless Sensor Networks[J].Journal of Interconnection Networks 2007, Vol. 8, No. 4, pp.355-367.

[2] K. C. Jeongyeup, K. Chintalapudi, J. Paek et al, Embedded Sensing of Structures: A Reality Check[R]. RTCSA 2014,pp.95-101.

[3] Y. S. Jeong, Y. C. Hwang, et al, Efficient cluster-based routing protocol for wireless sensor network[R]. WSEAS Transactions on Communications, 2009.5,v5,n5,pp.868-876.

[4] A. Mellouk, S. Hoceiniet al, Design and performance analysis of an Conductive QoS routing algorithm [J]. Computer Communications,2009.7,v32,n12,pp.1371-1376.

[5] http://www.chinawsn.com.cn/article/WSN/luyou/2008-06-04/29.html, 2010-05.

[6] Information onhttp://www.bianjibu.net/tongxin/220.html, 2010-02. 